UCSB-HEP-94-03

UMD-PP-94-90

February,1994

\title{
Accommodating solar and atmospheric neutrino deficits, hot dark matter, and a double beta decay signal
}

\author{
David O. Caldwell* \\ Department of Physics, University of California, Santa Barbara, California 93106 \\ Rabindra N. Mohapatra+Supported in part by the National Science Foundation \\ Department of Physics, University of Maryland, College Park, Maryland 20742
}

\begin{abstract}
Neutrino mass explanations of the solar and atmospheric neutrino deficits and a hot dark matter component require one of three patterns of those masses, as already pointed out by us. Recently there have been indications of a non-vanishing amplitude for neutrinoless double beta decay. If this additional hint of neutrino mass is true, it would make even less likely the one unfavored pattern (a sterile neutrino giving warm, rather than hot dark matter), would alter another by making the $\nu_{e}$ a contributor to the hot dark matter, and would make the third $\left(\nu_{e}, \nu_{\mu}\right.$, and $\nu_{\tau}$ approximately degenerate) much more likely than previously. For this third case we construct a gauge model consistent with other weak interaction data. This model utilizes a more general version of the see-saw mechanism, which is very likely to be the source of neutrino mass, if this degenerate pattern is correct. A new supernova constraint is utilized, and implications and tests of the different mass matrices are noted.

* Supported in part by the Department of Energy
\end{abstract}




\section{INTRODUCTION}

There are several different observations involving neutrinos which receive a plausible and satisfactory explanation if the neutrinos are massive, which they are not in the Standard Model. First is the well-known solar neutrino deficit [1], observed by four different experiments [2]. Second is the deficit of muon neutrinos relative to electron neutrinos produced in the atmosphere, as measured by three experiments [3]. Third is the likely need for a neutrino component of the dark matter of the universe to understand the structure and density on all distance scales [4]. We showed [5] that, consistent with particle physics and cosmological constraints, there are only three conceivable patterns of neutrino mass which could explain these three phenomena. Of the three, one gave warm dark matter, rather than the favored hot component, and one other also appeared dubious for Majorana masses because of the limitation on electron neutrino mass from neutrinoless double beta decay, $\beta \beta_{0 \nu}$.

The $\beta \beta_{0 \nu}$ situation has now changed, however, and leads us to emphasize this formerly less favored pattern of neutrino masses. What was previously [6] a limit on effective neutrino mass has, after another year and a half of data taking, become about a twostandard-deviation indication for that mass [7]. With current matrix element calculations, this effective Majorana mass from the enriched ${ }^{76} \mathrm{Ge}$ experiment is $\left\langle m_{\nu}\right\rangle \sim 1-2 \mathrm{eV}$. Adding interest to this possibility is the observation in a ${ }^{130} \mathrm{Te}$ experiment [8] of a similar twostandard-deviation effect. In this case the even more uncertain matrix element calculations would favor $\left\langle m_{\nu}\right\rangle \sim 4 \mathrm{eV}$. Clearly much more experimental work is needed before this hint of neutrino mass is even at the level of believability of the three mentioned above, but the uncertainties in the nuclear matrix elements make it possible that the ${ }^{76} \mathrm{Ge}$ and ${ }^{130} \mathrm{Te}$ experiments are observing the same real effect. In case this is true, we wish to point out how all of these results could be accommodated theoretically.

First, however, we review the constraints on neutrino mass from experiments, cosmology, and astrophysics.

\section{INPUT INFORMATION}

\section{A. Solar Neutrino Deficit}

For massive neutrinos which can oscillate from one species to another, the solar electron neutrino observations [2] can be understood if the neutrino mass differences and mixing angles fall into one of the following ranges [9], where the Mikheyev-Smirnov-Wolfenstein (MSW) mechanism is included [10]:

$$
\text { a)Small - angle MSW, } \Delta m_{e i}^{2} \sim 6 \times 10^{-6} \mathrm{eV}^{2}, \sin ^{2} 2 \theta_{e i} \sim 7 \times 10^{-3},
$$




$$
\begin{aligned}
& \text { b) Large - angle MSW, } \Delta m_{e i}^{2} \sim 9 \times 10^{-6} \mathrm{eV}^{2}, \sin ^{2} 2 \theta_{e i} \sim 0.6 \text {, } \\
& \text { c) Vacuum oscillation, } \Delta m_{e i}^{2} \sim 10^{-10} \mathrm{eV}^{2}, \sin ^{2} 2 \theta_{e i} \sim 0.9 .
\end{aligned}
$$

Of these, (a) is favored over (b) by the fits to the solar neutrino data [9], and both (b) and (c) are disfavored by information from the neutrino burst from supernova 1987A [11].

\section{B. Atmospheric Neutrino Deficit}

The second set of experiments indicating non-zero neutrino masses and mixings has to do with atmospheric $\nu_{\mu}$ 's and $\nu_{e}$ 's arising from the decays of $\pi$ 's and $K$ 's and the subsequent decays of secondary muons produced in the final states of the $\pi$ and $K$ decays. In the underground experiments the $\nu_{\mu}$ and $\bar{\nu}_{\mu}$ produce muons and the $\nu_{e}$ and $\bar{\nu}_{e}$ lead to $e^{ \pm}$. Observations of $\mu^{ \pm}$and $e^{ \pm}$indicate a far lower value for $\nu_{\mu}$ and $\bar{\nu}_{\mu}$ than suggested by naïve counting arguments which imply that $N\left(\nu_{\mu}+\bar{\nu}_{\mu}\right)=2 N\left(\nu_{e}+\bar{\nu}_{e}\right)$. More precisely, the ratio of $\mu$ events to $e$-events can be normalized to the ratio of calculated fluxes to reduce flux uncertainties, giving [3]

$$
\begin{aligned}
R(\mu / e) & =0.60 \pm 0.07 \pm 0.05 \text { (Kamiokande) } \\
& =0.54 \pm 0.05 \pm 0.12(\mathrm{IMB}) \\
& =0.69 \pm 0.19 \pm 0.09(\text { Soudan II })
\end{aligned}
$$

Combining these results with observations of upward going muons by Kamiokande [3], IMB [3], and Baksan [12] and the negative Fréjus [13] and NUSEX [14] results leads to the conclusion [15] that neutrino oscillations can give an explanation of these results, provided

$$
\Delta m_{\mu i}^{2} \approx 0.005 \text { to } 0.5 \mathrm{eV}^{2}, \sin ^{2} 2 \theta_{\mu i} \approx 0.5 \text {. }
$$

\section{Hot Dark Matter}

There is increasing evidence that more than $90 \%$ of the mass in the universe must be detectable so far only by its gravitational effects. This dark matter is likely to be a mix of $\sim 30 \%$ of particles which were relativistic at the time of freeze-out from equilibrium in the early universe (hot dark matter) and $\sim 70 \%$ of particles which were non-relativistic (cold dark matter). Such a mixture [16] gives the best fit [4] of any available model to the structure and density of the universe on all distance scales, such as the anisotropy of the microwave background, galaxy-galaxy angular correlations, velocity fields on large and small scales, correlations of galaxy clusters, etc. A very plausible candidate for hot dark matter is one or more species of neutrinos with total mass of $m_{\nu_{H}}=93 h^{2} F_{H} \Omega=7 \mathrm{eV}$, if $h=0.5$ (the Hubble constant in units of $100 \mathrm{~km} \cdot \mathrm{s}^{-1} \cdot \mathrm{Mpc}^{-1}$ ), $F_{H}=0.3$ (the fraction 
of dark matter which is hot), and $\Omega=1$ (the ratio of density of the universe to closure density). We shall use the frequently quoted $7 \mathrm{eV}$ below, but different determinations give $h=0.45 \pm 0.09$ [17] or $h=0.80 \pm 0.11$ [18] (a value giving difficulties with $\Omega=1$ ), making $m_{\nu_{H}}=2$ or $21 \mathrm{eV}$. However, $F_{H}$ is probably less than 0.3 , since a baryonic content of $F_{B}=(0.010-0.015) / h^{2}$ must be accommodated and $F_{H}=0.2$ is not unlikely [19].

It is usually assumed that the $\nu_{\tau}$ would supply the hot dark matter. This is justified on the basis of an appropriately chosen see-saw model [20] and a $\nu_{e} \rightarrow \nu_{\mu}$ MSW explanation of the solar $\nu$ deficit. However, if the atmospheric $\nu_{\mu}$ deficit is due to $\nu_{\mu} \rightarrow \nu_{\tau}$, the $\nu_{\tau}$ alone cannot be the hot dark matter, since the $\nu_{\mu}$ and $\nu_{\tau}$ need to have close to the same mass. It is interesting that instead of a single $\sim 7 \mathrm{eV}$ neutrino, sharing that $\sim 7 \mathrm{eV}$ between two or among three neutrino species provides a better fit to the universe structure and particularly a better understanding of the variation of matter density with distance scale $[21]$.

\section{Nucleosynthesis Limits on Neutrino Species}

While the $Z^{0}$ width limits the number of weakly interacting neutrino species to three, the nucleosynthesis limit [22] of about 3.3 on the number of light neutrinos is more useful here, since it is independent of the neutrino interactions. Invoking a fourth neutrino, $\nu_{s}$, which is sterile, meaning it does not have the usual weak interaction, must be done with parameters such that it will not lead to overproduction of light elements in the early universe. For example, the atmospheric $\nu_{\mu}$ problem cannot be explained by $\nu_{\mu} \rightarrow \nu_{s}$, since $\sin ^{2} 2 \theta_{\mu s} \approx 0.5$ is too large for the $\Delta m_{\mu s}^{2}$ involved, and that $\nu_{s}$ would have been brought into equilibrium in the early universe [23]. On the other hand, the solar $\nu_{e}$ problem can be explained by $\nu_{e} \rightarrow \nu_{s}$ for either the small-angle MSW or the vacuum oscillation solutions, but not for the less favored large-angle MSW solution [23].

\section{E. Neutrinoless Double Beta Decay}

As mentioned in the Introduction, there are indications from the Heidelberg-Moscow ${ }^{76} \mathrm{Ge}$ experiment [7] and the Milan ${ }^{130} \mathrm{Te}$ experiment [8] that there may be an effective Majorana neutrino mass of

$$
\left\langle m_{\nu}\right\rangle \sim 1-2 \mathrm{eV} .
$$

In terms of individual neutrino masses $m_{\nu_{i}}$ and mixing matrix elements $U_{i j}$,

$$
\left\langle m_{\nu}\right\rangle \approx\left|\sum_{i} \eta_{i} U_{e i}^{2} m_{\nu_{i}}\right|
$$

where $\eta_{i}= \pm 1$, depending on the CP property of the individual neutrino. Thus there may 
be a cancellation in the sum, making it possible that the $\left\langle m_{\nu}\right\rangle$ which $\beta \beta_{0 \nu}$ can measure is much smaller than the actual $m_{\nu_{e}}$. We shall address this issue below.

\section{POSSIBLE PATTERNS OF NEUTRINO MASS}

A. Patterns Required by Solar and Atmospheric Neutrino Deficits and Hot Dark Matter

With the above input information, if the solar neutrino puzzle is resolved by either $\nu_{e} \rightarrow \nu_{\mu}$ or $\nu_{e} \rightarrow \nu_{s}$ oscillations, the atmospheric neutrino deficit is due to $\nu_{\mu} \rightarrow \nu_{\tau}$ oscillations, and some hot dark matter is required, then there are only three possible textures for neutrino masses, as we have pointed out [5]:

(i). All three neutrinos are nearly degenerate, with $m_{\nu_{e}} \approx m_{\nu_{\mu}} \approx m_{\nu_{\tau}} \approx 2-3 \mathrm{eV}$, since $\nu_{e} \rightarrow \nu_{\mu}$ and $\nu_{\mu} \rightarrow \nu_{\tau}$ both require small mass differences, but the required dark matter mass can be shared.

(ii). The three neutrinos with weak interactions are light, and a sterile neutrino supplies the dark matter, but the early decoupling required to satisfy the nucleosynthesis constraint reduces the number density of the $\nu_{s}$, forcing the mass of $\nu_{s}$ to be so large that it becomes warm, rather than the desired hot, dark matter.

(iii). The $\nu_{e}$ and $\nu_{s}$ can be quite light to take care of the solar neutrino problem while the $\nu_{\mu}$ and $\nu_{\tau}$ share the dark matter role, being $\sim 3-4 \mathrm{eV}$ each, and explain the atmospheric $\nu_{\mu}$ deficit.

B. Added Effect of a Non-Zero Neutrinoless Double Beta Decay Amplitude

The primary purpose of this paper is to discuss the impact on the neutrino mass textures worked out in Ref. [5] for the above three cases should the indications for an effective Majorana mass for the neutrino, $\left\langle m_{\nu}\right\rangle \sim 1-2 \mathrm{eV}$, be confirmed [24]. First, however, it is worth pointing out that there could then be no quadratic or linear see-saw model justification for the $\nu_{\tau}$ alone to be hot dark matter.

If convincing peaks are seen at the appropriate energies for more than one $\beta \beta$ isotope, and $\left\langle m_{\nu}\right\rangle$ is determined within the uncertainties of the nuclear matrix elements, there still remains the issue of possible cancellations in Eq. (4) in trying to extract the mass of $\nu_{e}$ (or more precisely, $\left.\nu_{1}\right)$. As was pointed out in [5], for case (i) the unitarity of the mixing matrix limits the one undetermined mixing angle, that of $\nu_{e}-\nu_{\tau}$, to 0.05 for the small-angle MSW solution. The smallness of the $\nu_{e}-\nu_{\tau}$ and $\nu_{e}-\nu_{\mu}$ angles and hence of the mixing matrix elements $U_{e 2}$ and $U_{e 3}$ makes $\left\langle m_{\nu}\right\rangle \approx m_{\nu_{1}} \approx m_{\nu_{e}}$ for this case. This conclusion is true also for the large-angle MSW and vacuum oscillation solutions, since the near mass degeneracy of the $\nu_{1}, \nu_{2}$, and $\nu_{3}$ forces the $\nu_{2}$ and $\nu_{3}$ terms in Eq. (4) to nearly cancel, or else $\beta \beta_{0 \nu}$ would have been observed long ago. 
For case (iii), as in case (i), the same considerations would apply for the small-angle MSW solution, since the $\nu_{s}$ has to be very weakly mixed to satisfy the nucleosynthesis bound. Nucleosynthesis also eliminates the large-angle MSW solution and in the vacuum oscillation case forces the $\nu_{s}$ to be mixed strongly only with the $\nu_{e}$. In this last instance (case (iii), vacuum oscillations), a limitation on the effect of the $\nu_{\mu}$ and $\nu_{\tau}$ masses may be invoked from supernovae considerations. If, as appears likely, the heavy elements in the universe are produced by a rapid neutron capture process in the supernova environment, then the $\nu_{e}-\nu_{\mu}$ and $\nu_{e}-\nu_{\tau}$ mixing angles are severely restricted $\left(\sin ^{2} 2 \theta \lesssim 4 \times 10^{-4}\right)$ for $\Delta m^{2} \gtrsim 4 \mathrm{eV}^{2}$ (with a rapid decrease in $\sin ^{2} 2 \theta$ for larger $\Delta m^{2}$ ) [25]. Otherwise the energetic $\nu_{\mu}$ and $\nu_{\tau}(\langle E\rangle \approx 25 \mathrm{MeV})$ can convert to $\nu_{e}$ 's which have much higher energy than the thermal $\nu_{e}$ 's $(\langle E\rangle \approx 11 \mathrm{MeV})$. The higher energy $\nu_{e}$ 's, having a larger cross section, will reduce the neutron density via $\nu_{e}+n \rightarrow e^{-}+p$, diminishing heavy element formation. With the effect of the $\nu_{\mu}$ and $\nu_{\tau}$ eliminated in Eq. (4) and $\nu_{e}$ and $\nu_{s}$ of approximately equal mass, the pseudo-Dirac (opposite $\mathrm{CP}$ eigenvalues) combination is not allowed, since $\left\langle m_{\nu}\right\rangle \approx 0$, whereas in the case of the same CP eigenvalues, $m_{\nu_{e}} \approx\left\langle m_{\nu}\right\rangle / 2$.

The added $\beta \beta_{0 \nu}$ constraint makes case (ii) even less likely but now for a different reason. Since the $\nu_{e}, \nu_{\mu}$, and $\nu_{\tau}$ could provide much, or even all, of the hot dark matter, there is little or no reason to invoke a sterile neutrino. We will not deal any further with this unlikely possibility.

In case (iii), the new information alters the allowed range of parameters. For example, $\nu_{e}$ and $\nu_{s}$, recalling the uncertainty in the $\beta \beta_{0 \nu}$ nuclear matrix elements, could be $\sim 1$ $\mathrm{eV}$ each, but only the $\nu_{e}$ would contribute to dark matter. The $\nu_{s}$ must decouple early (probably even before the quark-hadron phase transition at $T \sim 200 \mathrm{MeV}$ ), in order not to contribute excessively (i.e., $\delta N_{\nu} \leq 0.3$ [22]) to the energy density of the universe at the epoch of nucleosynthesis. Using the nominal $7 \mathrm{eV}$, the $\nu_{\mu}$ and $\nu_{\tau}$ would then be $\sim 3$ $\mathrm{eV}$ each, and the most likely way to determine that this pattern of masses is correct is to observe $\nu_{\mu} \rightarrow \nu_{e}$ oscillations for $\Delta m^{2} \approx 8 \mathrm{eV}^{2}$, such as could be done in the current LSND experiment at Los Alamos, perhaps requiring some uncertainty in the supernova constraint [25] just discussed. While $\beta \beta_{0 \nu}$ is the only currently feasible way to determine the masses in scheme (i), and $\nu_{\mu} \rightarrow \nu_{e}$ oscillations plus $\beta \beta_{0 \nu}$ are the way to get masses in scheme (iii), the choice between these two alternatives could be made on the basis of whether the solar $\nu_{e}$ deficit is due to $\nu_{e} \rightarrow \nu_{\mu}$ or $\nu_{e} \rightarrow \nu_{s}$. The measurement of the charged to neutral current ratio by the Sudbury Neutrino Observatory distinguishes $\nu_{e} \rightarrow \nu_{\mu}$ from $\nu_{e} \rightarrow \nu_{s}$, but proving the latter (as opposed to an astrophysical cause of the $\nu_{e}$ deficit) 
requires a measurement of spectral distortions $[9,26]^{*}$.

$$
\text { C. Mass Matrix for Case (iii) }
$$

The generic form of the Majorana mass matrix for case (iii), as given in Ref. [5], in the basis $\left(\nu_{s}, \nu_{e}, \nu_{\mu}, \nu_{\tau}\right)$,

$$
M=\left(\begin{array}{cccc}
\mu_{1} & \mu_{3} & \epsilon_{11} & \epsilon_{12} \\
\mu_{3} & \mu_{2} & \epsilon_{21} & \epsilon_{22} \\
\epsilon_{11} & \epsilon_{21} & m & \delta / 2 \\
\epsilon_{12} & \epsilon_{22} & \delta / 2 & m+\delta
\end{array}\right)
$$

For simplicity, we set the $\epsilon_{i j}=0$, and we shall demonstrate later that those terms are indeed negligible for most purposes. The implication of the $\beta \beta_{0 \nu}$ constraint is that $\mu_{1}+$ $\delta_{1}=\mu_{2}=1-2 \mathrm{eV}$. For the small-angle MSW case, $\mu_{1} \gg \delta_{1} \gg \mu_{3} \approx 5 \times 10^{-8} \mathrm{eV}$, and $\delta_{1} \approx 1.5 \times 10^{-6} \mathrm{eV}$. For the vacuum oscillation case, $2 \mu_{3} \approx \delta_{1} \approx 10^{-10} \mathrm{eV}$. Recall that the large-angle MSW and the pseudo-Dirac $\nu_{e}-\nu_{s}$ vacuum oscillation cases are ruled out. A total neutrino mass of the nominal $7 \mathrm{eV}$ implies that $m \approx 2 \mathrm{eV}$, and $\delta \approx 10^{-1}-10^{-3} \mathrm{eV}$, which is a slight change from the values of the parameters given in Ref. [5].

Returning now to the $\epsilon_{i j}$, if the $\epsilon_{i j} \ll \mu_{1,2}$ (or $\mu_{3}$ ) and $m$, then they perturb the eigenvalues only slightly, but they lead to mixings between $\nu_{\mu}$ and $\nu_{\tau}$ with $\nu_{s}$. These $\nu_{\mu}-\nu_{s}$ and $\nu_{\tau}-\nu_{s}$ mixings are severely constrained by nucleosynthesis [23], implying that $\epsilon_{i j} \lesssim 10^{-5}-10^{-6} \mathrm{eV}$, since $\Delta m^{2} \sim$ few $e V^{2}$. Somewhat weaker constraints also result from the supernova argument [25] given above, which leads to $\epsilon_{2 j} \lesssim 10^{-3} \mathrm{eV}$, since $\theta_{e j} \approx \epsilon_{2 j} / m$. It is, therefore, justifiable to neglect $\epsilon_{i j}$, except for determining the mixings.

\section{Mass Matrices for Case (i)}

We turn now to the case of three highly degenerate neutrino eigenstates, which will be the favored scenario, if future $\beta \beta_{0 \nu}$ results yield a mass large enough to account for a third of the hot dark matter mass. Since all the values of $\Delta m_{i j}^{2}$ in this case are small (i.e., $\lesssim 0.5$ $\mathrm{eV}^{2}$ ), the mixing angles are not constrained by heavy element production by supernovae [25]. In a subsequent section, we will present a gauge model which will provide this mass degeneracy in a "natural" manner (i.e., without any arbitrary adjustment of parameters), guaranteed by a horizontal symmetry.

Here and in Ref. [5] our conclusions about favored neutrino mass textures have been based on the assumption of two-flavor neutrino oscillations. The solar and atmospheric

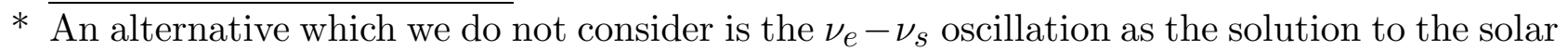
neutrino deficit but $\nu_{\mu}-\nu_{e}$ oscillation for the atmospheric case by making $\nu_{e}$ slightly heavier than $\nu_{\mu}$; the $\nu_{\tau}$ would then be the main contributor to hot dark matter. This is unlikely because the observed absolute $\nu_{e}$ flux as a function of energy agrees with calculations. Note that if $m_{\nu_{e}} \simeq 2 e V$, this becomes like case (iii) with a rather bizzare mass ordering. 
neutrino data can be treated together using a three-flavor oscillation scenario, but only the recent work by Kim and Lee [27] could be made compatible with the need for hot dark matter and $\left\langle m_{\nu}\right\rangle \sim 2 \mathrm{eV}$, although they did not consider this possibility. They assumed that all three neutrinos mix maximally and found mass differences compatible with the two-flavor oscillation values given in Eq. (1) for vacuum oscillations and in Eq. (2) above. Their assumption of maximal mixing requires a different mass matrix than that given in Ref. [5] for the three-degenerate-neutrino case. Both possibilities are given below in forms compatible with solar and atmospheric neutrino data, a Majorana $m_{\nu_{e}} \sim 2 \mathrm{eV}$, and total mass sufficient for hot dark matter.

Case A. The mass matrix in the $\nu_{e}, \nu_{\mu}, \nu_{\tau}$ basis is the same as in Ref. [5], since it corresponds to two-flavor oscillations:

$$
M=\left(\begin{array}{ccc}
m+\delta_{1} s_{1}^{2} & -\delta_{1} c_{1} c_{2} s_{1} & -\delta_{1} c_{1} s_{1} s_{2} \\
-\delta_{1} c_{1} c_{2} s_{1} & m+\delta_{1} c_{1}^{2} c_{2}^{2}+\delta_{2} s_{2}^{2} & \left(\delta_{1}-\delta_{2}\right) c_{2} s_{2} \\
-\delta_{1} c_{1} s_{1} s_{2} & \left(\delta_{1}-\delta_{2}\right) c_{2} s_{2} & m+\delta_{1} s_{2}^{2}+\delta_{2} c_{2}^{2}
\end{array}\right)
$$

where $c_{i}=\cos \theta_{i}$ and $s_{i}=\sin \theta_{i}, m=2 \mathrm{eV} ; \delta_{1} \simeq 1.5 \times 10^{-6} \mathrm{eV} ; \delta_{2} \simeq .2$ to $.002 \mathrm{eV}$; $s_{1} \simeq 0.05 ;$ and $s_{2} \simeq 0.4$ for the small-angle MSW solution. Note that it is a somewhat more accurate version of the Eq. (1) in Ref. [5]. For the large-angle MSW or vacuum oscillation solutions, $\delta, s_{1}$, and $s_{2}$ will be different.

Case B. The mass matrix in the same basis but for three-flavor oscillations and maximal mixings [27] is

$$
M=\left(\begin{array}{ccc}
m+\delta_{1}+\delta_{2} & -x \delta_{1}-x^{2} \delta_{2} & -x^{2} \delta_{1}-x \delta_{2} \\
-x^{2} \delta_{1}-x \delta_{2} & m+\delta_{1}+\delta_{2} & x \delta_{1}+x^{2} \delta_{2} \\
-x \delta_{1}-x^{2} \delta_{2} & x^{2} \delta_{1}+x \delta_{2} & m+\delta_{1}+\delta_{2}
\end{array}\right)
$$

where $m=2 \mathrm{eV}, \delta_{1}=\frac{1}{4} \times 10^{-10} \mathrm{eV}, \delta_{2}=\frac{1}{4}\left(10^{-2}-10^{-3}\right) \mathrm{eV}$, and $x=e^{2 \pi i / 3}$, now for the case of vacuum oscillations only.

In comparing cases $\mathrm{A}$ and $\mathrm{B}$, we see that in case $\mathrm{A}$, except for the high degree of degeneracy of the diagonal terms, the other entries are hierarchical but not related to each other. That is, elements $M_{12} \ll M_{13} \ll M_{23} \ll M_{i i}$. On the other hand, in case B, all non-diagonal elements are the same to leading order and all diagonal entries are exactly the same; i.e., $M_{11}=M_{22}=M_{33}$ and $\left|M_{i j}\right|=\delta_{2}$ for $i \neq j$. Case B, therefore, will be much harder to obtain in a natural manner for a gauge model than Case A. For this reason, and because the vacuum oscillation solution is much less likely, in the next section we present a gauge model which reproduces a mass matrix of the type in Case A only. 


\section{DEGENERATE MAJORANA NEUTRINOS FROM GRANDUNIFICATION}

Before discussing details of the model, we note some important points about any gauge model that could reproduce the mass matrix of case A, Eq. (6). First, the high degree of degeneracy of the neutrino masses, and no evidence for such degeneracy elsewhere in the fermion spectrum, implies that the dominant masses for neutrinos must arise from a different source than that of quarks and charged leptons. This feature is present in see-saw type models, as we show below. Second, there must be a horizontal symmetry, $G_{H}$, in the neutrino sector, and the minimal $G_{H}$ (whether discrete or continuous) must have a three-dimensional representation. Third, since the horizontal symmetry is broken in the quark and charged lepton masses, one expects corrections to the neutrino degeneracy from them, as well as any possible horizontal symmetry breaking effects in the neutrino sector. The smallness of the neutrino degeneracy breaking terms must be stable under radiative corrections.

Below we present an $\mathrm{SO}(10)$ grandunified model which can lead to the desired pattern of neutrino masses without arbitrary fine tuning of parameters, but first we give the qualitative reason this works. In the early days of the discussion of the see-saw formula for neutrino masses, it was pointed out [28] that implementing that mechanism in the simplest left-right or $\mathrm{SO}(10)$ models resulted in a $\nu_{L^{-}} \nu_{R}$ mass matrix of the modified see-saw form:

$$
\nu_{L}\left(\begin{array}{cc}
\nu_{L} & \nu_{R} \\
\nu_{R} & m_{\nu_{D}} \\
m_{\nu_{D}}^{T} & f v_{R}
\end{array}\right),
$$

where $f$ and $m_{\nu_{D}}$ are $3 \times 3$ matrices and $v_{L} \approx \lambda M_{W_{L}}^{2} / v_{R}$. The light neutrino mass matrix that follows from diagonalizing Eq. (7) is

$$
m_{\nu} \approx f \lambda M_{W_{L}}^{2} / v_{R}-m_{\nu_{D}} f^{-1} m_{\nu_{D}}^{T} / v_{R}+\ldots
$$

While both terms vanish as $v_{R} \rightarrow \infty$, the first term always dominates over the second one for neutrino masses. This negates the usual quadratic formula (i.e., the second term) for neutrino masses. It means that usual see-saw quadratic mass formula is not realized in generic left-right or $\mathrm{SO}(10)$ models, unless additional assumptions are made, as has been pointed out [29] (e.g., decoupling the parity and $\mathrm{SU}(2)_{R}$ scales in left-right symmetric models, or in the singlet Majoron models, etc.).

Accepting the more complex see-saw formula given above, it is clear that if a symmetry dictates that $f_{a b}=f \delta_{a b}$, then the neutrino masses are degenerate to leading order. For 
$v_{R} \approx 10^{13.5} \mathrm{GeV}, f \lambda \approx 1$, we get $m_{\nu_{e}}=m_{\nu_{\mu}}=m_{\nu_{\tau}} \approx 1.5 \mathrm{eV}, m_{\nu_{\mu}}^{2}-m_{\nu_{e}}^{2} \approx 3 m_{c}^{2} / 10 f v_{R} \approx$ $10^{-4} / f \mathrm{eV}^{2}$, and $m_{\nu_{\tau}}^{2}-m_{\nu_{\mu}}^{2} \approx 3 m_{t}^{2} / 10 f v_{R} \approx(2 / f)\left(m_{t} / 150 \mathrm{GeV}\right)^{2} \mathrm{eV}^{2}$. These mass differences are of the right order of magnitude to explain the solar neutrino (via the MSW mechanism) and the atmospheric neutrino puzzles, while the masses roughly give the dark matter.

It is also interesting to note that the B-L breaking scale of $v_{R} \sim 10^{13} \mathrm{GeV}$ emerges naturally from constraints of $\sin ^{2} 2 \theta_{W}$ and $\alpha_{s}$ in non-supersymmetric $\mathrm{SO}(10)$ grandunified theories [30], enhancing the reason for an $\mathrm{SO}(10)$ scenario. To guarantee the neutrino degeneracy (i.e., $f_{a b}=f \delta_{a b}$ ), an extra $\mathrm{SU}(2)_{H}$ family symmetry [31] is imposed on the model. This family symmetry will be broken softly by terms in the Lagrangian of dimension two, so that departures from the degeneracy in the neutrino sector are naturally small.

Turning now to some details of the model, the known fermions of each family are assigned as usual to a $\{16\}$-dimensional spinor representation of $\mathrm{SO}(10)$, and we denote them by $\psi_{a}$ ( $a=$ family index, going over $\left.1,2,3\right)$. We assume that the $\psi_{a}$ transform as a triplet under $\mathrm{SU}(2)_{H}$, which is taken as a softly broken global family symmetry. As to the Higgs sector, the $\mathrm{SO}(10)$ symmetry is assumed to break down at the GUT scale, $M_{U}$, to the left-right symmetric group $\mathrm{SU}(2)_{L} \times \mathrm{SU}(2)_{R} \times \mathrm{SU}(4)_{C} \times P \equiv G_{224 P}$ via the non-zero vev of a $\{54\}$-dimensional scalar multiplet. We wish to emphasize that the existence of parity as a good local symmetry below the GUT scale is important for the more general see-saw formula, (8), to work [29]. The $G_{224 P}$ symmetry is broken down to the Standard Model at a scale $v_{R}$ by a $\{126\}$-dimensional Higgs multiplet, denoted by $\Delta_{0}$. Since $\left\langle\Delta_{0}\right\rangle$ breaks B-L symmetry, it gives the heavy Majorana mass to the right-handed neutrino. The $\Delta_{0}$ is assumed to be an $\mathrm{SU}(2)_{H}$ singlet in order to guarantee the $f$ matrix in Eq. (7) to be an identity matrix (see later).

We assume that there are six complex $\{10\}$-dimensional Higgs multiplets denoted by $H_{a b}$, (with $H_{a b}=H_{b a}$, where $a, b=1,2,3$ ) which transform like a $\{1\}+\{5\}$-dimensional representation under $\mathrm{SU}(2)_{H}$ symmetry. We denote the singlet by $\operatorname{Tr} H$ and the $\{5\}$ by $H^{5}$. Two Standard Model doublets in each H-multiplet acquire vev's at the electroweak scale and are denoted by $\kappa_{a b}^{u}$ and $\kappa_{a b}^{d}$. In order to get a realistic charged fermion spectrum (and avoid disastrous relations such as $m_{s}=m_{\mu}$ at $M_{U}$ ), we need five more $\{126\}$-dimensional multiplets denoted by $\Delta_{a b}$ (with $\Delta_{a b}$ symmetric and traceless in the indices $a$ and $b$ ), which acquire induced vev's of order the electroweak scale. We keep the (mass) ${ }^{2}$ of $\Delta_{a b}$ to be positive and of order the GUT scale, so that they do not break B-L symmetry and thus do not contribute to Majorana masses. 
The part of the Higgs potential that is responsible for the weak scale vev's, as well as the B-L breaking vev is

$$
\begin{aligned}
V_{1}^{0} & =-\mu_{a b}^{2} H_{a b}^{5 \dagger} H_{a b}^{5}-\tilde{\mu}_{a b}^{2} H_{a b} H_{a b}+\lambda_{1}\left(\operatorname{Tr} H^{\dagger} H\right)^{2}+\lambda_{2} \operatorname{Tr} H^{\dagger} H H^{\dagger} H+\lambda_{3} \operatorname{Tr} H^{\dagger} H H^{\dagger} \operatorname{Tr} H \\
& +\lambda_{R}\left(\Delta_{0}^{\dagger} \Delta_{0}-v_{R}^{2}\right)^{2}+M_{a b}^{2} \Delta_{a b}^{\dagger} \Delta_{a b}+\lambda_{3}\left(\operatorname{Tr} \Delta^{\dagger} \Delta\right)^{2}+\lambda_{4} \operatorname{Tr}\left(\Delta^{\dagger} \Delta \Delta^{\dagger} \Delta\right) \\
& +\beta_{1} \Delta_{0}^{\dagger} \Delta_{0} \Delta_{a b} H_{a b}+\beta_{2} \Delta_{0}^{\dagger} \Delta_{0} \Delta_{0} \operatorname{Tr} H+\gamma \Delta_{0} \Delta_{0} \operatorname{Tr}(H H)+\text { h.c. }
\end{aligned}
$$

(In writing this potential, we have assumed a softly broken Peccei-Quinn (PQ) symmetry, as in Ref. [32].) The $\lambda_{R}$ term generates the $\Delta_{0}$-vev which breaks the B-L symmetry and also breaks $G_{224 P}$ down to the Standard Model. The terms $\beta_{1}$ and $\beta_{2}$ generate the induced weak-scale vev's for the $(2,2,15)$ components of both $\Delta_{0}$ and $\Delta_{a b}$; we denote these vev's by $v_{a b}^{u}$ and $v_{a b}^{d}$, respectively. This induced vev mechanism [32] has the advantage that it avoids any need for extra fine tunings of the $\Delta$ masses. In fact, in this model only the masses of $H_{a b}$ need to be fine tuned to the electroweak scale. Note that the $\gamma$ term in the above equation generates the $v_{L}$ term in $\Delta_{0}$, which causes the approximate neutrino degeneracy.

The $\mathrm{SO}(10) \times \mathrm{SU}(2)_{H}$-invariant Yukawa coupling is given by

$$
\mathcal{L}_{Y}=h \psi_{a} \psi_{b} H_{a b}+f_{0} \psi_{a} \psi_{a} \bar{\Delta}_{0}+f_{1} \psi_{a} \psi_{b} \bar{\Delta}_{a b}+\text { h.c. }
$$

The softly broken PQ symmetry prevents the coupling of $H^{*}$ to fermions.

The resulting charged fermion mass matrix becomes

$$
\begin{aligned}
M_{u, a b} & =h \kappa_{a b}^{u}+f_{0} v_{0}^{u} \delta_{a b}+f_{1} v_{a b}^{u} \\
M_{d, a b} & =h \kappa_{a b}^{d}+f_{0} v_{0}^{d} \delta_{a b}+f_{1} v_{a b}^{d} \\
M_{\ell, a b} & =h \kappa_{a b}^{d}-3 f_{0} v_{0}^{d} \delta_{a b}-3 f_{1} v_{a b}^{d} \\
M_{\nu D} & =h \kappa_{a b}^{u}-3 f_{0} v_{0}^{u} \delta_{a b}-3 f_{1} v_{a b}^{u} .
\end{aligned}
$$

The neutrino mass matrix has the desired form given in Eq. (6). Since there are 23 free parameters in the mass matrices, it is not surprising that the quark masses and mixings and charged lepton masses can be reproduced easily. It is worth emphasizing that, even though the particle spectra of the model may appear quite dense, the low energy spectrum consists only of the particles of the Standard Model, along with massive neutrinos.

This model has a prediction for the lifetime of the proton, which can be obtained from Ref. [30]. Ignoring heavy-particle threshold corrections and QCD coupling uncertainties, one gets $\tau_{P} \approx 10^{33}$ years, which would be reachable by Super Kamiokande. The existence 
of so many heavy particles, however, is likely to introduce an uncertainty by one or two orders of magnitude.

If future experiments bear out a degenerate light neutrino spectrum, this detailed and quite complicated $\mathrm{SO}(10)$ model may not be the appropriate description of the physics. Its essential ingredient, the correct general see-saw formula, [8], will almost surely be required to fit those data, however.

\section{CONCLUSIONS}

The solar and atmospheric neutrino data, along with a need for some hot dark matter, if all are due to neutrino mass, limit the texture of those masses to one of three possibilities, probably only two of which seem viable. The third alternative, under the conditions of Ref. [5], had a sterile neutrino, $\nu_{s}$, which gave warm dark matter. If further neutrinoless double beta decay, $\beta \beta_{0 \nu}$, experiments confirm a Majorana mass for the $\nu_{e}$ around $2 \mathrm{eV}$ or so, the $\nu_{s}$ could become superfluous. A $\nu_{e}$ mass found by $\beta \beta_{0 \nu}$ would contribute to the hot dark matter in the viable alternative scheme in which the solar problem is solved by $\nu_{e} \rightarrow \nu_{s}$, but the main contribution would come from the $\nu_{\mu}$ and $\nu_{\tau}$, with $\nu_{\mu} \rightarrow \nu_{\tau}$ solving the atmospheric $\nu_{\mu}$ problem. The final alternative has the hot dark matter mass shared almost equally among $\nu_{e}, \nu_{\mu}$, and $\nu_{\tau}$, with the solar neutrino deficit now being $\nu_{e} \rightarrow \nu_{\mu}$. This last scheme becomes particularly attractive if $\nu_{e}$ is $\sim 2 \mathrm{eV}$, and so we have presented a gauge model that reproduces this degenerate neutrino spectrum without affecting the vastly nondegenerate masses for charged leptons. Should this be the true pattern of neutrino mass, even if this model is not the correct description, its basis in a version of the see-saw mechanism which is more generally correct than that usually invoked is very likely to be the source of those masses.

\section{REFERENCES}

[1]. J.N. Bahcall and M.H. Pinsonneault, Rev. Mod. Phys. 64, 885 (1992).

[2]. R. Davis et al., Proceedings of the 21st International Cosmic Ray Conference, Vol. 12, edited by R.J. Protheroe (Univ. of Adelaide Press, Adelaide, 1990), p. 143; K.S. Hirata et al., Phys. Rev. D 44, 2241 (1991); A.I. Abrazov et al., Phys. Rev. Lett. 67, 3332 (1991) and V.N. Gavrin in TAUP 93 Workshop, Gran Sasso, Italy, 1993 (unpublished);

P. Anselmann et al., Phys. Lett. B285, 376 (1992) and B314, 445 (1993).

[3]. K.S. Hirata et al., Phys. Lett. B280, 146 (1992); R. Becker-Szendy et al., Phys. Rev. D 46, 3720 (1992); P.J. Litchfield in International Europhysics Conference on High Energy Physics, Marseille, France, 1993 (unpublished). 
[4]. E.L. Wright et al., Astrophys. J. 396, L13 (1992); M. Davis, F.J. Summers, and D. Schagel, Nature 359, 393 (1992); A.N. Taylor and M. Rowan-Robinson, ibid. 359, 396 (1992); R.K. Schaefer and Q. Shafi, Report No.BA-92-28, 1992 (unpublished) and Nature 359, 199 (1992); J.A. Holtzman and J.R. Primack, Astrophys. J. 405, 428 (1993); A. Klypin et al., Astrophys. J. 416, 1 (1993).

[5]. D.O. Caldwell and R.N. Mohapatra, Phys. Rev. D 48, 3259 (1993).

[6]. A. Balysh et al., Phys. Lett. B283, 32 (1992).

[7]. A. Piepke in International Europhysics Conference on High Energy Physics, Marseille, France, 1993 (unpublished).

[8]. E. Garcia in TAUP 93 Workshop, Gran Sasso, Italy, 1993 (unpublished).

[9]. Of the many analyses, we use the recent results of N. Hata and P. Langacker, Report No. UPR-0592T, 1993 (to be published in Phys. Rev.) and P.I. Krastev and S.T. Petcov, Report No. SISSA 177/93/EP, 1993 (unpublished).

[10]. S.P. Mikheyev and A.Yu. Smirnov, Yad. Fiz. 42, 1441 (1985); L. Wolfenstein, Phys. Rev. D 17, 2369 (1978); 20, 2634 (1979).

[11]. A.Yu. Smirnov, D.N. Spergel, and J.N. Bahcall, Institute for Advanced Study Report No. IASSNS-AST 93/15, 1993 (to be published in Phys. Rev. D).

[12]. M.M. Boliev et al. in Proceedings of the 3rd International Workshop on Neutrino Telescopes, Venice, Italy, 1991, edited by M. Baldo-Ceolin (Istitute Nazionale di Fisica Nucleare, Padova, 1991), p. 235.

[13]. Ch. Berger et al., Phys. Lett. B245, 305 (1990); 227, 489 (1989).

[14]. M. Aglietta et al., Europhys. Lett. 15, 559 (1991).

[15]. W. Frati et al., Phys. Rev. D 48, 1140 (1993).

[16]. Q. Shafi and F.. Stecker, Phys. Rev. Lett. 53, 1292 (1984).

[17]. A. Sandage et al., Astrophys. J. 401, L7 (1992).

[18]. G.H. Jacoby et al., PASP 104, 599 (1992).

[19]. U. Seljak and E. Bertschinger, MIT Report MIT-CSR-93-33, 1993 (unpublished).

[20]. M. Gell-Mann, P. Ramond, and R. Slansky in Supergravity, edited by D. Freedman et al. (1979); T. Yanagida, in KEK Lectures, edited by O. Sawada et al. (1979); R. Mohapatra and G. Senjanović, Phys. Rev. Lett. 44, 912 (1980). 
[21]. M. Davis and J. Primack (private communications); Y.P. Ying et al. Univ. of Arizona Report 93-0590, 1993 (to be published in Astronomy and Astrophysics).

[22]. T.P. Walker et al., Astrophys. J. 376, 51 (1991).

[23]. P. Langacker, Univ. of Pennsylvania Report No. UPR 0401T, 1989 (unpublished); R. Barbieri and A. Dolgov, Nucl. Phys. B349, 743 (1991); K. Enqvist, K. Kainulainen, and J. Maalampi, Phys. Lett. B249, 531 (1990); M.J. Thomson and B.H.J. McKellar, Phys. Lett. B259, 113 (1991); V. Barger et al., Phys. Rev. D 43, 1759 (1991); P. Langacker and J. Liu, Phys. Rev. D 46, 4140 (1992); X. Shi, D. Schramm, and B. Fields, Phys. Rev. D 48, 2563 (1993); J. Cline, Phys. Rev. Lett. 68, 3137 (1992).

[24]. For a recent discussion of $\beta \beta_{0 \nu}$ and just the solar neutrino problem, see S.T. Petcov and A.Yu. Smirnov, Report No. SISSA 113/93/EP, 1993 (unpublished).

[25]. Y.-Z. Qian et al., Phys. Rev. Lett. 71, 1965 (1993).

[26]. S.M. Bilenky and C. Giunti, University of Torino Report No. DFTT 62/93, 1993 (unpublished).

[27]. C.W. Kim and J.A. Lee, Johns Hopkins Univ. Report No. JHU-TIPAC-930023, 1993 (unpublished).

[28]. R.N. Mohapatra and G. Senjanović, Phys. Rev. D 23, 165 (1981).

[29]. D. Chang and R.N. Mohapatra, Phys. Rev. D 32, 1248 (1985).

[30]. R.N. Mohapatra and G. Senjanović, Z. Phys. 17, 53 (1983); N.G. Deshpande, R. Keith, and P.B. Pal, Phys. Rev. D 46, 2261 (1992).

[31]. After this work was done, we were informed that a degenerate neutrino spectrum from $\mathrm{SU}(2)_{H}$ symmetry in the context of low-scale electroweak models has been discussed by K.S. Babu and S. Pakvasa (private communication).

[32]. K.S. Babu and R.N. Mohapatra, Phys. Rev. Lett. 70, 2845 (1993). 